\title{
Mortalidade de idosos em município do Sudeste brasileiro de 2006 a 2011
}

\author{
Mortality of the elderly in a municipality of southeastern Brazil \\ from 2006 to 2011
}

Rodolpho Telarolli Júnior ${ }^{1}$

Leonor de Castro Monteiro Loffredo ${ }^{2}$

${ }^{1}$ Departamento de Ciências Biológicas, Faculdade de Ciências Farmacêuticas de Araraquara, Universidade Estadual Paulista Júlio de Mesquita Filho (UNESP). Rodovia Araraquara-Jaú km 1, Campus Universitário. 14.800-901 Araraquara SP Brasil.

rtelarollijr@terra.com.br ${ }^{2}$ Departamento de Odontologia Social, Faculdade de Odontologia, UNESP.
Abstract This paper addressed the mortality rate for elderly people in Araraquara in the state of São Paulo between 2006 and 2011. An epidemiological descriptive study was conducted using data from the National Mortality Information System and the Data Analysis State System Foundation. The ratio between mortality rates by point $(R)$ and by $95 \%$ confidence interval $\left(I C_{95 \%}\right)$ were estimated. More than $60 \%$ of elderly people had low education, and $76 \%$ of them died in hospital. For the period from 2006 to 2008 a statistically significant difference was observed between males and females, the most common causes of death being circulatory disease $R=1.41\left(I C_{95 \%}: 1.24\right.$ $1.58)$, respiratory problems $R=1.49\left(I C_{95 \%}: 1.22\right.$ $1.76)$, and cancer $R=1.79\left(I_{95 \%}: 1.40-2.18\right)$. For the period from 2009 to 2011, circulatory diseases accounted for $R=1.18$ ( $\left.\left.I C_{95 \%}: 1.03-1.33\right)\right]$, and the differences were significant for respiratory disease $R=1.33\left(I_{95 \%}: 1.11-1.55\right)$ and cancer $R=$ $1.94\left(I C_{95 \%}: 1.53-2.35\right)$. The fourth and fifth more frequent causes of death among the elderly population were diabetes mellitus and external causes, respectively. The pattern of mortality found emphasizes the importance of actions aimed at reducing the major causes of death such as increasing the coverage of the influenza vaccine and control of hypertension and diabetes mellitus.

Key words Elderly, Mortality, Descriptive epidemiology
Resumo O objetivo foi descrever a mortalidade entre idosos em Araraquara (SP), no período de 2006 a 2011. Estudo epidemiológico descritivo, tendo como fontes de dados o Sistema de Informações sobre Mortalidade e a Fundação Sistema Estadual de Análise de Dados. Foi calculada razão entre coeficientes de mortalidade por ponto $(R)$ e por intervalo de $95 \%$ de confiança $\left(I C_{95 \%}\right)$. Observou-se mais de $60 \%$ dos idosos com nivel baixo de escolaridade, sendo que $76 \%$ faleceram em hospitais. Entre 2006 e 2008, as diferenças foram estatisticamente significantes entre homens e mulheres, predominando as doenças circulatórias com $R=1,41$ ( IC $\left._{95 \%}: 1,24-1,58\right)$, respiratórias com $R=1,49$ $\left(I C_{95 \%}: 1,22-1,76\right)$ e neoplasias com $R=1,79\left(I_{95 \%}\right.$ : 1,40-2,18). Entre 2009 e 2011, obteve-se, para as causas circulatórias $R=1,18$ ( IC $_{95 \%}: 1,03-1,33$ ), sendo significativas as diferenças para as respiratórias com $R=1,33\left({ }_{1} C_{95 \%}: 1,11-1,55\right)$ e câncer sendo $R=1,94$ ( $\left.I C_{95 \%}: 1,53-2,35\right)$. O diabetes mellitus e as causas externas apareceram, respectivamente, como quarta e quinta causas de mortes mais frequentes na população idosa. O padrão de mortalidade encontrado ressalta a importância de ações voltadas à redução das principais causas de morte, como o incremento da cobertura da vacina contra a influenza e o controle da hipertensão arterial e do diabetes mellitus.

Palavras-chave Idoso, Mortalidade, Epidemiologia descritiva 
Introdução

O rápido envelhecimento da população brasileira nos últimos 50 anos é, por suas causas e consequências, um dos processos de mudança demográfica mais significativos verificados na história do país. Mas foi apenas a partir da década de 1980, com a realização de um grande número de estudos em várias áreas da ciência, que a questão da transição demográfica brasileira, com todas as suas implicações para a sociedade, tornou-se pauta obrigatória nos centros de pesquisa ${ }^{1}$.

O envelhecimento da população é um processo inexorável que vem ocorrendo em todo o mundo, datando do século XIX e primeira metade do século XX nos países da Europa e América do Norte. Já nos países emergentes esse é um processo mais recente, da segunda metade do século XX. $O$ certo é que, uma vez iniciado o conjunto de mudanças demográficas e sociais que levam ao envelhecimento de uma população, provavelmente este seja um caminho sem retorno. Para 2030, algumas estimativas apontam para $21,4 \%$ de indivíduos com 65 anos e mais na Europa, 11,2\% na América Latina e Caribe, $10,7 \%$ na Ásia e apenas 4,6\% na África, continente onde a transição demográfica ainda caminha lentamente ${ }^{2}$.

No Brasil, o envelhecimento populacional vem ocorrendo de forma desigual. Os dados do censo de 2010 mostram o país com uma média de $10,8 \%$ de habitantes com 60 anos e mais. Quando os números são distribuídos por regiões, encontram-se 6,9\% de idosos na região Norte, $8,8 \%$ no Centro-Oeste, $10,3 \%$ no Nordeste, $11,8 \%$ no Sudeste e $12,0 \%$ na região $\mathrm{Sul}^{3}$. O envelhecimento brasileiro acelerou-se na segunda metade do século XX: enquanto em 1900 os dados do censo mostravam 3,2\% de indivíduos com 60 anos e mais, esse índice passou para 4,2\% em 1920, mantendo-se nesse patamar até 1950. A partir do recenseamento de 1960 a participação dos idosos na população geral aumentou progressivamente, com 4,7\% naquele ano, 5,1\% em 1970 , $6,1 \%$ em $1980,7,7 \%$ em 1991, 8,6\% em 2000, chegando aos 10,8\% em 2010 3 .

A região Central do Estado de São Paulo é uma das que vem envelhecendo mais rapidamente, tendo sido objeto de estudos anteriores ${ }^{4}$. O município de Araraquara situa-se nessa região de recentes transformações socioeconômicas, tendo como principais atividades a agroindústria da cana e da laranja, a indústria de tecnologia, além da prestação de serviços e o ensino superior. Para 2011 a Fundação Sistema Estadual de Análise de Dados (SEADE) estimou um total de 13,9\% de habitantes com 60 anos e mais para Araraquara, enquanto a média do Estado de São Paulo era de $11,5 \%{ }^{5}$. Para 2020 são estimados $15,4 \%$ de moradores do Estado de São Paulo com 60 anos e mais; nessa data, estimam-se $18,4 \%$ de pessoas nessa faixa etária vivendo no município de Araraquara, quando os maiores de 60 anos em Araraquara corresponderão ao triplo das crianças de até 4 anos, os idosos superando, pela primeira vez, os menores de 15 anos $^{5}$.

Assim, é de grande importância o estudo das variáveis relacionadas ao envelhecimento nessa região do Estado de São Paulo, que, por suas características econômicas e sociais, acaba antecipando condições demográficas e de saúde que serão vivenciadas pelo restante do Estado daqui a alguns anos.

Este estudo tem por objetivo caracterizar a mortalidade da população com 60 anos e mais do município de Araraquara, São Paulo, no período de 2006 a 2011, segundo as variáveis causa básica, local de ocorrência do óbito e escolaridade, nos dois sexos. Com isso, esperamos contribuir com a elaboração de políticas de saúde que atendam às prioridades sanitárias da população idosa dessa região do Estado de São Paulo.

\section{Métodos}

Os dados de mortalidade de indivíduos com 60 anos e mais foram obtidos a partir do Sistema de Informação de Mortalidade da Secretaria de Vigilância Epidemiológica do Ministério da Saúde.

O município de Araraquara situa-se na região Central do Estado de São Paulo, com uma população estimada para $2011 \mathrm{em} 211.247$ habitantes, encontrando-se na categoria dos que tem valor máximo de IPRS (Índice Paulista de Responsabilidade Social), que corresponde a uma adaptação à realidade do Estado de São Paulo do IDH - Índice de Desenvolvimento Humano, proposto pelo Programa das Nações Unidas para o Desenvolvimento (PNUD) ${ }^{5}$.

As estimativas de população por sexo, idade e escolaridade foram obtidas do Instituto Brasileiro de Geografia e Estatística (IBGE) ${ }^{3}$ e da Fundação SEADE 5 .

No tocante à variável causa, trabalhou-se com causas escolhidas de morte, aquelas que têm apresentado maior prevalência em estudos epidemiológicos realizados anteriormente $e^{6,7}$ como as neoplasias, as doenças do aparelho circulatório, as doenças do aparelho respiratório, o diabetes e as causas externas segundo o CID-10 (10 ${ }^{a}$ Classifi- 
cação Internacional de Doenças). Entre as doenças infecciosas e parasitárias, que no conjunto provocam poucas mortes na faixa etária, foram discriminadas as mortes por aids e as hepatites. O destaque para essas duas causas de morte infecciosas deve-se ao incremento da sua prevalência, no país, em pessoas de 60 anos e mais ${ }^{8}$.

Foi feita estatística descritiva dos dados de mortalidade, sendo analisados a escolaridade, o local de ocorrência e a causa para o período de 2006 a 2011 em residentes no município de Araraquara. Adicionalmente, o período de 2006 a 2011 foi subdividido (2006-2008 e 2008-2011), para análise dos coeficientes específicos de mortalidade por causa (x 100.000). Para a comparação de dois coeficientes segundo causa entre os sexos, calculou-se o intervalo de $95 \%$ de confiança $\left(\mathrm{IC}_{95 \%}\right)$ para a razão entre eles $(\mathrm{R})$, julgandose como estatisticamente não significante o intervalo que incluísse o valor $1^{9}$.

\section{Resultados}

Em Araraquara, em 2010, a população era composta por $48,2 \%$ de homens e $51,8 \%$ de mulhe- res. Na faixa etária de 60 anos e mais, $42,2 \%$ eram homens e $57,8 \%$ mulheres. Viviam na zona rural 2,8\% da população geral e 9,8\% dos idosos de Araraquara.

Entre 2006 e 2011 ocorreram 5.763 óbitos de pessoas com 60 anos e mais, sendo 2.921 do sexo feminino e 2.842 do sexo masculino. Quanto à distribuição percentual dos óbitos dos idosos segundo sexo, até 2009 ela manteve-se equilibrada, valendo, em média, 50\%. Porém, a partir de 2010 verificou-se valores percentuais diferentes, valendo 52,5 e 47,0, respectivamente ao sexo feminino e masculino.

O grau de escolaridade pode ser observado nas Figuras 1 e 2, referentes ao sexo masculino e feminino, respectivamente.

Para o sexo masculino predominaram, em todo o período, os indivíduos com até 3 anos de escolaridade, seguidos por aqueles com 4 a 7 anos de escolaridade (ensino básico incompleto). Um resultado que chamou a atenção foi o avanço de pessoas com 12 anos e mais de estudo (ensino superior completo e incompleto), que passou de $3,9 \%$ para $9,9 \%$, um aumento superior a $250 \%$ no índice de indivíduos com maior escolaridade. Os homens idosos analfabetos tiveram sua par-
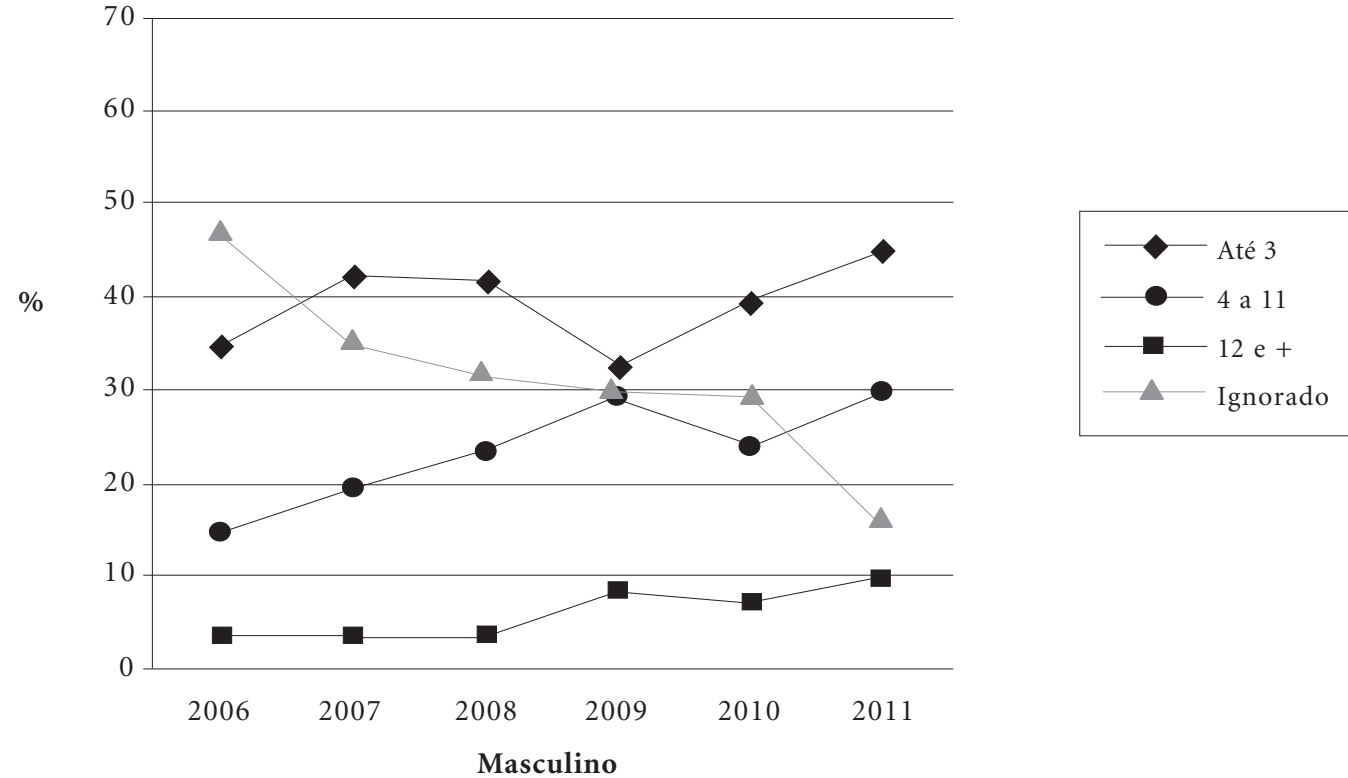

Figura 1. Distribuição percentual dos óbitos de idosos, entre 2006 e 2011, no sexo masculino, segundo escolaridade, em Araraquara, SP. 


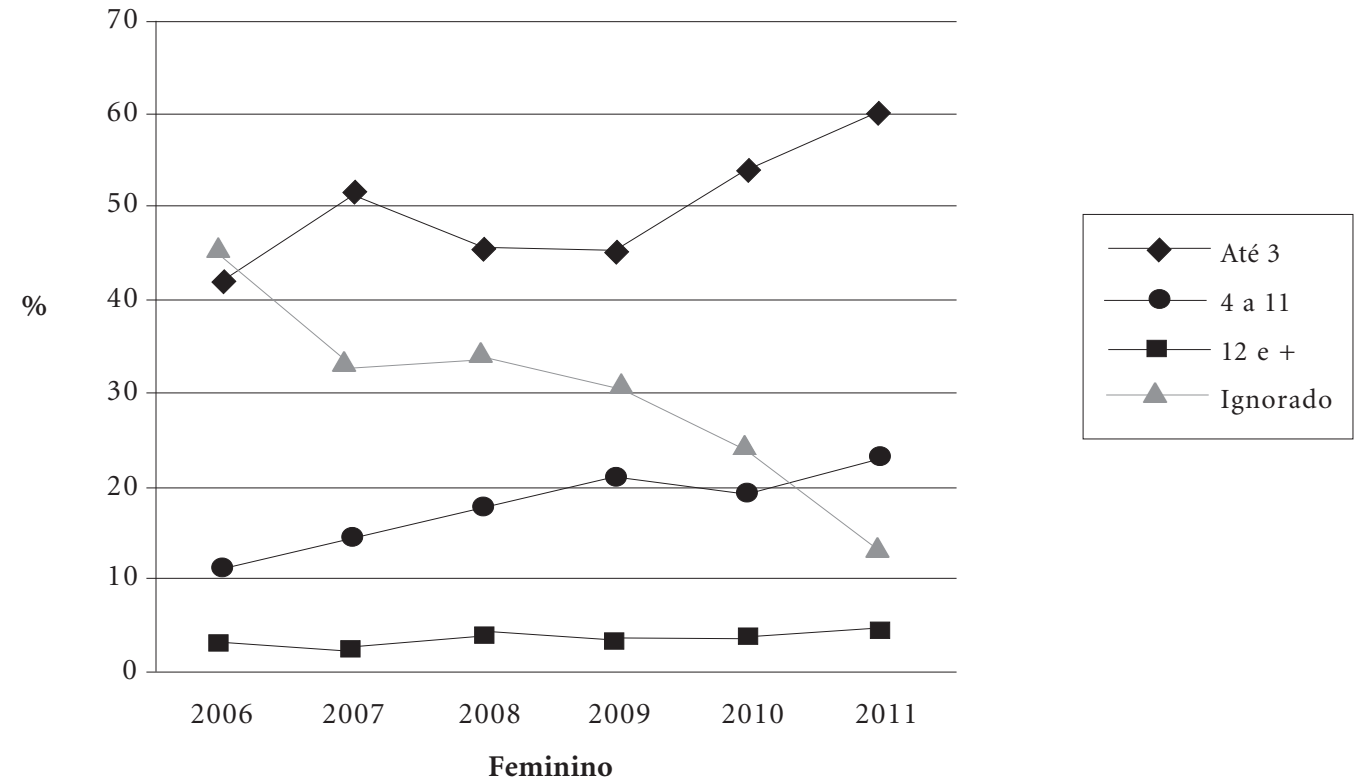

Figura 2. Distribuição percentual dos óbitos de idosos, entre 2006 e 2011, no sexo feminino, segundo escolaridade, em Araraquara, SP.

Fonte: SIM, Ministério da Saúde, $2011^{10}$.

ticipação reduzida em $32,2 \%$ no período, de $6,8 \%$ em 2006 para 4,6\% em 2011.

Entre os óbitos do sexo feminino os resultados se repetiram, acentuando-se as tendências já ressaltadas para o sexo masculino, conforme se observa na Figura 2.

A distribuição percentual dos óbitos de indivíduos com 60 anos e mais, segundo escolaridade, mostrou os homens com um patamar mais alto de escolaridade do que as mulheres.

Quanto ao local, 76\% dos óbitos ocorreram em hospital, 20\% em domicílio e $4 \%$ em outros estabelecimentos de saúde ou na rua, ao longo do período. Mesmo sendo pouco frequentes, observou-se que a ocorrência de morte em via pública é 10 vezes mais frequente no sexo masculino, sendo que $0,7 \%$ dos óbitos do sexo masculino ocorreram em via pública enquanto, para mulheres, foi de $0,07 \%$. Para os dois sexos, foi observado um aumento nas mortes ocorridas em outros estabelecimentos de saúde, os asilos e as casas de repouso. Entre os homens as mortes ocorridas nesses locais aumentaram $246 \%$, passando de 1,3\% em 2006 para 3,2\% em 2011. Para as mulheres o aumento foi de $264 \%$, os óbitos dessa categoria passando de 1,1\% em 2006 para 2,9\% em 2011.

Quanto às causas de morte, as mais comuns foram, em ordem decrescente, as decorrentes de doenças do aparelho circulatório, do aparelho respiratório, das neoplasias, o diabetes mellitus, as mal definidas e as causas externas, conforme os dados da Tabela 1.

As doenças do aparelho circulatório foram as mais comuns no período 2006 a 2011, apresentando uma tendência de diminuição para homens e mulheres, com uma redução na participação do total de mortes de $20,4 \%$ e $9,2 \%$, respectivamente ao sexo masculino e feminino, ao longo do período.

Entre os homens as causas circulatórias mais comuns foram: doença cérebro-vascular (10,5\%), infarto agudo do miocárdio $(9,4 \%)$, insuficiência cardíaca $(3,9 \%)$, outras doenças isquêmicas do coração $(3,4 \%)$ e miocardiopatias $(2,6 \%)$. Entre as mulheres as causas circulatórias mais comuns foram: doença cérebro-vascular (10,7\%), infarto agudo do miocárdio $(6,8 \%)$, insuficiência cardíaca $(5,5 \%)$, doença hipertensiva $(3,7 \%)$ e miocardiopatias $(3,4 \%)$. 
Tabela 1. Distribuição percentual dos óbitos de indivíduos com 60 anos e mais, entre 2006 e 2011, residentes no município de Araraquara, SP, segundo sexo (M: masculino; F: feminino) e principais causas de morte.

\begin{tabular}{|c|c|c|c|c|c|c|c|}
\hline \multirow[b]{2}{*}{ Causa } & \multirow[b]{2}{*}{ Sexo } & \multicolumn{6}{|c|}{$\%$} \\
\hline & & 2006 & 2007 & 2008 & 2009 & 2010 & 2011 \\
\hline \multirow[t]{2}{*}{ Aparelho circulatório } & M & 37,7 & 33,1 & 37,3 & 32,6 & 33,1 & 30,0 \\
\hline & $\mathrm{F}$ & 39,2 & 34,3 & 31,6 & 34,6 & 34,5 & 35,6 \\
\hline \multirow[t]{2}{*}{ Aparelho respiratório } & M & 13,4 & 19,8 & 18,9 & 17,7 & 18,5 & 19,0 \\
\hline & $\mathrm{F}$ & 12,8 & 13,8 & 20,7 & 19,1 & 16,8 & 17,7 \\
\hline \multirow[t]{2}{*}{ Neoplasias } & M & 13,4 & 11,7 & 13,5 & 16,6 & 10,3 & 12,9 \\
\hline & $\mathrm{F}$ & 10,4 & 10,4 & 8,5 & 6,9 & 10,1 & 10,8 \\
\hline \multirow[t]{2}{*}{ Diabetes mellitus } & M & 2,8 & 4,4 & 2,4 & 3,1 & 3,3 & 4,0 \\
\hline & $\mathrm{F}$ & 4,4 & 4,5 & 4,8 & 4,2 & 5,5 & 4,6 \\
\hline \multirow[t]{2}{*}{ Mal definidas } & M & 1,8 & 2,3 & 2,4 & 1,6 & 2,0 & 1,6 \\
\hline & $\mathrm{F}$ & 3,8 & 3,8 & 3,5 & 4,5 & 3,9 & 3,5 \\
\hline \multirow[t]{2}{*}{ Externas } & M & 3,5 & 2,5 & 3,5 & 2,6 & 2,9 & 3,2 \\
\hline & F & 2,9 & 2,3 & 2,4 & 2,3 & 5,1 & 1,1 \\
\hline \multirow[t]{2}{*}{ Outras } & M & 30,2 & 30,6 & 24,4 & 28,9 & 33,2 & 33,3 \\
\hline & F & 30,9 & 35,4 & 33,3 & 32,6 & 29,6 & 31,3 \\
\hline
\end{tabular}

Fonte: SIM, Ministério da Saúde, $2011^{10}$.

Entre as causas circulatórias, a doença hipertensiva foi $46,4 \%$ mais comum entre mulheres que entre homens na média do período, com índices de $2,5 \%$ e $3,7 \%$, respectivamente para os sexos masculino e feminino. Já o infarto agudo do miocárdio foi $37,6 \%$ mais comum entre homens que entre mulheres no período, com valores, respectivamente, de $9,4 \%$ e 6,8\%. Já a insuficiência cardíaca mostrou-se $43,4 \%$ mais comum como causa de morte entre as mulheres, com índices de 3,9\% para o sexo masculino e 5,5\% para o sexo feminino.

As doenças respiratórias tiveram sua participação aumentada no período, com uma elevação no período de $41,8 \%$ e $38,3 \%$, respectivamente entre homens e mulheres. Esse aumento da participação das causas respiratórios deu-se, basicamente, às custas das mortes por pneumonias. Entre os homens as mortes por pneumonias passaram de $7,0 \%$ para $13,1 \%$ no período 2006 2011 (incremento de $87,1 \%$ ), enquanto entre as mulheres essas mortes foram de $9,9 \%$ para $13,3 \%$ no mesmo período (incremento de 34,3\%).

As neoplasias malignas predominaram entre os homens, em todos os anos do período analisado, destacando-se as de pulmão $(3,0 \%)$, próstata $(2,2 \%)$ e cólon, reto e ânus $(2,1 \%)$. Para as mulheres, foram mais comuns as de cólon, reto e ânus $(2,2 \%)$, mama $(2,2 \%)$ e pulmão $(1,5 \%)$.
Em 2011, o diabetes mellitus, como causa de morte entre homens, aumentou 42,8\%, passando de 2,8\% (2006) para 4,0\% (2011). Entre as mulheres manteve-se estável no período.

As causas externas, para todos os anos do período, predominaram entre os homens, com exceção de 2010, chamando a atenção o alto índice observado entre as mulheres (5,1\%). Em média, no período, as mortes decorrentes de quedas foram $67 \%$ mais comuns entre as mulheres que os homens, com índices de 2,1\% e 1,2\%, respectivamente. Já as mortes decorrentes de acidentes de trânsito foram $122 \%$ mais frequentes entre homens $(0,9 \%)$ que mulheres $(0,4 \%)$, enquanto as mortes por outros acidentes, homicídios e suicídios foram 4 vezes mais comuns no sexo masculino, valendo, em média, 0,9\% entre homens e $0,2 \%$ entre mulheres, no período estudado.

Merece destaque nos resultados encontrados a pequena participação das causas mal definidas, com 2,0\% para os homens e 3,8\% para as mulheres, no período.

Em relação à aids e às hepatites infecciosas, registram-se poucos casos na população em estudo. Foram apenas 3 óbitos tendo como causa básica o vírus HIV, em 2006, 2009 e 2010. Em relação às hepatites, contabilizaram-se 9 óbitos em todo o período, sendo 7 por hepatite B, correspondendo a $0,1 \%$ do total de mortes. 
Os coeficientes específicos por causas foram calculados por triênios, nos dois sexos, podendo ser visualizados na Tabela 2 .

Para o período 2006-2008, verificou-se diferença estatisticamente significante entre sexos para as doenças circulatórias $\left(\mathrm{IC}_{95 \%}: 1,24-1,58\right)$, doenças respiratórias $\left(\mathrm{IC}_{95 \%}: 1,22-1,76\right)$ e neoplasias $\left(\mathrm{IC}_{95 \%}: 1,40-2,18\right)$. Comportamento análogo foi observado para o período seguinte, 2009 2011, sendo que, para as causas circulatórias a razão entre os sexos permaneceu próxima ao limite de significância $\left(\mathrm{IC}_{95 \%}: 1,03-1,33\right)$. Já para o diabetes mellitus e para as causas externas essas diferenças foram estatisticamente não significantes, ou seja, os coeficientes de mortalidade foram semelhantes nos dois sexos, nos dois períodos.

Constatou-se ainda que, para as causas circulatórias entre os homens, houve decréscimo no coeficiente de 2006-2008 ( $\mathrm{C}=1519,97 \mathrm{x}$ 100.000) para o período 2009-2011 ( $\mathrm{C}=1268,35$ $\mathrm{x} 100.000)$, sendo a diferença estatisticamente significante, pois $\mathrm{R}=1,20\left(\mathrm{IC}_{95 \%}: 1,05-1,35\right)$.

\section{Discussão}

Este estudo procurou analisar a tendência da mortalidade entre idosos de Araraquara (SP), tomando como fonte de dados o SIM, que é o Sistema de Informações de Mortalidade implantado em 1975 pelo Ministério da Saúde, com abrangência nacional. É um sistema de vigilância epidemiológica confiável que se presta a disponibilizar as informações de saúde a partir das declarações de óbito ${ }^{11}$. Um parâmetro que permite avaliar a qualidade do preenchimento das declarações de óbito é o índice de causas básicas mal definidas, de forma que é possível inferir que quanto menor esse índice haverá uma qualidade superior desses documentos ${ }^{12}$. Entre 2006 e 2011, Araraquara apresentou 2,9\% de óbitos de idosos por causas mal definidas, valor muito inferior ao observado em 2007 no Rio de Janeiro (14,0\%), Porto Alegre (13,5\%), Belo Horizonte $(10,9 \%)$ e São Paulo $(10,6 \%)^{13}$.

Os coeficientes de mortalidade por sexo para maiores de 60 anos mostraram-se mais elevados para o sexo masculino em todo o período, acompanhando o padrão biológico geral, de maior mortalidade entre homens do que entre mulhe$\mathrm{res}^{14}$. Mas, ao mesmo tempo em que a longevi-

Tabela 2. Coeficientes específicos de mortalidade por causa (C x 100.000) de indivíduos com 60 anos e mais; razão entre os coeficientes por ponto $(\mathrm{R})$ e por intervalo de $95 \%$ de confiança $\left(\mathrm{IC}_{95 \%}\right)$. Períodos: 2006-2008 e 2009-2011.

\begin{tabular}{|c|c|c|c|c|c|}
\hline Causa & Período & Sexo & C (x100.000) & $\mathbf{R}$ & $\mathrm{IC}_{95 \%}$ \\
\hline Aparelho & $06-08$ & $\begin{array}{c}M \\
F\end{array}$ & $\begin{array}{l}1519,97 \\
1076,38\end{array}$ & 1,41 & $1,24-1,58$ \\
\hline Circulatório & $09-11$ & $\begin{array}{c}\mathrm{M} \\
\mathrm{F}\end{array}$ & $\begin{array}{l}1268,35 \\
1069,90\end{array}$ & 1,18 & $1,03-1,33$ \\
\hline Aparelho & $06-08$ & $\begin{array}{c}M \\
F\end{array}$ & $\begin{array}{l}719,83 \\
482,29\end{array}$ & 1,49 & $1,22-1,76$ \\
\hline Respiratório & $09-11$ & $\begin{array}{c}M \\
F\end{array}$ & $\begin{array}{l}724,38 \\
544,93\end{array}$ & 1,33 & $1,11-1,55$ \\
\hline \multirow[t]{2}{*}{ Neoplasias } & $06-08$ & $\begin{array}{c}\mathrm{M} \\
\mathrm{F}\end{array}$ & $\begin{array}{l}544,33 \\
304,72\end{array}$ & 1,79 & $1,40-2,18$ \\
\hline & $09-11$ & $\begin{array}{c}M \\
F\end{array}$ & $\begin{array}{l}554,90 \\
285,44\end{array}$ & 1,94 & $1,53-2,35$ \\
\hline \multirow[t]{2}{*}{ Diabetes Mellitus } & $06-08$ & $\begin{array}{c}M \\
F\end{array}$ & $\begin{array}{l}136,83 \\
144,69\end{array}$ & 1,06 & $0,66-1,46$ \\
\hline & $09-11$ & $\begin{array}{c}M \\
F\end{array}$ & $\begin{array}{l}136,67 \\
145,71\end{array}$ & 1,07 & $0,69-1,45$ \\
\hline \multirow[t]{2}{*}{ Causas externas } & $06-08$ & $\begin{array}{c}\mathrm{M} \\
\mathrm{F}\end{array}$ & $\begin{array}{r}130,88 \\
74,53\end{array}$ & 1,76 & $0,97-2,55$ \\
\hline & $09-11$ & $\begin{array}{c}\mathrm{M} \\
\mathrm{F}\end{array}$ & $\begin{array}{r}114,81 \\
85,83\end{array}$ & 1,34 & $0,77-1,91$ \\
\hline
\end{tabular}

Fonte: SIM, Ministério da Saúde, $2011^{10}$. 
dade feminina é maior, existe um maior comprometimento da qualidade de vida das idosas, com maior presença de doenças crônicas em mulheres, levando a uma autopercepção pior da própria saúde ${ }^{15}$.

Os dados de escolaridade dos óbitos com 60 anos e mais apresentaram padrões distintos para os sexos, com os homens mostrando mais anos de estudo que as mulheres, o que é compatível com um modelo social que ainda reserva menos oportunidades para estas, apesar de todos os avanços verificados nos últimos 50 anos. Geib ${ }^{16}$ observa que a desigualdade da escolaridade entre os sexos é apenas uma das muitas que afetam a população brasileira nesse indicador social. No Brasil, também se observam grandes desigualdades de escolaridade entre moradores do campo e da cidade, e entre as várias regiões do país. Um resultado que merece destaque nessa variável é a redução nos índices de óbitos com escolaridade desconhecida, o que leva a acreditar numa maior preocupação com o preenchimento dessa informação por parte de médicos.

No que diz respeito à variável local de ocorrência dos óbitos, encontrou-se 20\% de mortes em domicílio, um índice superior ao da população em geral, que foi de 15,2\% em Araraquara e de $13,8 \%$ para a média do Estado de São Paulo em $2010^{5}$. A proporção de óbitos em domićlio em Araraquara foi próxima aos 19,5\% observados em Recife, entre 2004 e $2006^{17}$. O local de ocorrência do óbito é um indicador disponível no SIM ainda pouco explorado pelos especialistas, mas uma hipótese para explicar a maior ocorrência de mortes em domicílio em Araraquara é a de que se trate de opção de muitas famílias, já que o município não sofre com a falta de leitos hospitalares. Araraquara é um município com população mais envelhecida que a média estadual e nacional e entre os idosos é elevada a prevalência de doenças de natureza crônica e degenerativa, nas quais a internação hospitalar não agrega qualidade ao tratamento, não aumenta a sobrevida nem melhora a qualidade de vida do doente ${ }^{18}$. Assim, índices mais elevados de óbitos em domicílio poderiam indicar, na verdade, a escolha de muitas famílias por um fim mais humanizado para seus familiares idosos, hipótese cuja confirmação demanda estudos complementares. Esta é uma opção que envolve um elevado custo humano e material, implicando na oferta de cuidados paliativos em domicílio, por cuidadores profissionais ou por familiares que se prestem a essa atividade ${ }^{19}$. O que reforça a importância do sistema de saúde investir na formação e supervisão dos cuidadores domiciliares, uma classe de profissionais cuja demanda vem aumentando em função do número crescente de idosos portadores de doenças crônicas e degenerativas que são mantidos em domicílio.

$\mathrm{O}$ índice de pessoas que morrem em casas de repouso em Araraquara foi de 3,8\% em 2010, próximo à média do Estado de São Paulo. Ainda é reduzido o número dos que vivem em casas de repouso no Brasil, pois as residências de longa permanência para idosos são mal vistas pela população, que associa esse tipo de moradia ao abandono e à exclusão social. Mas cada vez mais essa é uma alternativa que tende a ser adotada por muitos, como resultado das mudanças decorrentes da transição demográfica, que inviabilizam o cuidado dos idosos exclusivamente por familiares ${ }^{6}$. As causas de morte mais comuns, no período, foram as mesmas para os dois sexos: em primeiro lugar, com $34,7 \%$ das mortes, as causas circulatórias, seguidas pelas respiratórias $(17,4 \%)$, as neoplasias $(10,7 \%)$ e o diabetes mellitus $(4,1 \%)$. No conjunto, essas causas responderam por $65,9 \%$ das mortes no sexo masculino e $68,7 \%$ das mortes no sexo feminino. Comparando-se os sexos, a participação relativa das causas externas foi maior no sexo masculino $(3,0 \%)$ que no feminino $(2,6 \%)$. Os números encontrados em Araraquara mostram-se diferentes aos de outras regiões do país: em Belo Horizonte (MG), em Porto Alegre (RS), no Rio de Janeiro (RJ) e em São Paulo (SP), em primeiro lugar apareceram, em 2007, as causas ligadas ao aparelho circulatório, seguidas pelas neoplasias e pelas doenças do aparelho respiratório ${ }^{20}$. Esse padrão também foi observado em Recife (PE), no período 2004-2006 ${ }^{17}$.

O padrão de mortalidade brasileiro é próximo ao dos Estados Unidos, com algumas especificidades. No Brasil é maior a mortalidade por doença cérebro-vascular e diabetes, enquanto nos Estados Unidos é maior a mortalidade por neoplasias e pneumonias ${ }^{21}$.

A queda nos coeficientes de mortalidade por causas circulatórias observadas em Araraquara acompanha o que tem sido uma tendência generalizada em todas as faixas de idade e regiões do país ${ }^{18}$. Essa redução generalizada na mortalidade por causas circulatórias tem sido a maior responsável pelo aumento da expectativa de vida ao nascer observada nas últimas décadas no Bra$\mathrm{sil}^{22}$. No Estado de Mato Grosso, observou-se tendência de declínio na mortalidade proporcional por doenças circulatórias e aumento nas causas respiratórias a partir da década de 1980, em 
estudo que também teve como fonte os números do $\mathrm{SIM}^{23}$. Entre os óbitos por causas circulatórias em Araraquara predominaram os acidentes vasculares cerebrais, nos dois sexos, padrão que é tendência em todo o Brasil, país que apresenta a segunda maior taxa de mortalidade por esta causa em todo o mundo ${ }^{7}$.

Além de principal causa de morte no país, as doenças do aparelho circulatório se constituem na principal causa de internação hospitalar entre os idosos ${ }^{7}$. Elas são uma das classes de doenças cujo tratamento mais onera o sistema de saúde. Em 2002, nos Estados Unidos, as cinco classes de doenças com o tratamento mais caro eram as doenças circulatórias, as neoplasias, os traumas, os distúrbios mentais e as doenças pulmonares ${ }^{24}$.

A mortalidade por causas respiratórias mostrou-se estável em Araraquara para o sexo masculino, tendo aumentado $13 \%$ entre as mulheres, comparando-se o primeiro ao segundo triênio do período em estudo. A mortalidade por doenças respiratórias é crescente à medida que a idade avança e no Brasil houve um aumento dos óbitos por essas causas de 30\% entre 1992 e 2005, mantendo-se sempre números mais elevados para o sexo masculino. Esse padrão parece estar relacionado ao tabagismo e a fatores relacionados às condições de trabalho ${ }^{25}$. Os resultados de mortalidade em idosos encontrados em Montes Claros (MG), situado em região de baixo desenvolvimento socioeconômico, parecem confirmar essa hipótese: em 2005 as doenças respiratórias não estavam sequer entre as três principais causas de mortes, sendo substituídas pelas doenças do Capítulo I da CID-10: Algumas doenças infecciosas e parasitárias ${ }^{26}$.

O aumento na proporção de óbitos decorrentes de causas respiratórias deu-se, basicamente, à custa das mortes por pneumonias. Estas mortes quase dobraram no sexo masculino (de $7,2 \%$, em 2006, para $13,1 \%$, em 2011) e aumentaram 20,9\% entre as mulheres (de 11,0\%, em 2006, para 13,3\%, em 2011). A baixa cobertura de vacinação contra a influenza certamente explica parte dessa tendência, já que no período 2006-2008, ela foi de apenas $66,8 \%$ no município de Araraquara, índice bem inferior aos 81,1\% observados na média do Estado de São Paulo ${ }^{27}$. Doenças relacionadas ao aparelho respiratório são a segunda principal causa de internação entre os idosos brasileiros ${ }^{28}$. A vacina é muito eficaz na prevenção da gripe e da pneumonia, reduzindo as internações hospitalares, tendo sido incorporada à rotina do SUS em 1999 na forma de campanhas anuais. A baixa adesão à vacina para a influenza é fato verificado em várias localidades, como Londrina (PR ${ }^{29}$ e Campinas (SP) ${ }^{30}$. As principais justificativas dos idosos para a não adesão à vacina é o medo de efeitos adversos e a falta de credibilidade na eficácia do produto, que também foi observado em idosos da Inglaterra ${ }^{31}$ e de outros países europeus ${ }^{32}$. Por ser uma atividade oferecida pelos serviços de saúde, é esperado que índices maiores de vacinação coincidam com serviços de saúde de melhor qualidade, tendo um papel importante a recomendação médica a favor da vacinação $0^{33}$.

Historicamente, tem havido um aumento na proporção de mortes por neoplasias em todas as regiões do país ${ }^{34}$. A mortalidade por neoplasias é multifatorial, relacionando-se ao estilo de vida, dieta, diagnóstico precoce e condições de vida, como observado no estudo do Recife $(\mathrm{PE})^{17}$. Indicador indireto, como morbidade hospitalar, também mostra aumento das neoplasias, como no estudo realizado em idosos do Rio de Janeiro $(\mathrm{RJ})^{7}$. Por se tratar de doença cuja incidência é mais elevada nas idades mais avançadas é de se esperar que o envelhecimento da população aumente a incidência de câncer em todo o país nas próximas décadas, como já vem sendo observado no Estado de São Paulo ${ }^{35}$ e nos Estados Unidos, onde a mortalidade na população idosa por neoplasias era $84,2 \%$ maior que a brasileira em $2000^{21}$.

A mortalidade por diabetes apareceu como a quarta causa mais comum de mortalidade de idosos em Araraquara, mantendo-se estável durante o período. Ao lado da hipertensão arterial sistêmica, o diabetes aparece como importante fator de risco para as doenças circulatórias, sendo fundamental o seu controle. De uma maneira geral, a ocorrência e a mortalidade por diabetes têm se mantido em níveis elevados em todo o país. Em 2006 o diabetes mellitus apareceu como doença autorreferida por $21,5 \%$ dos idosos de São Paulo $^{6}$ e, no ano seguinte, a ocorrência do diabetes, simultaneamente a outras doenças, multiplicou por 2,5 a chance de óbito do idoso ${ }^{36}$. Por problemas no controle da doença, no Brasil o diabetes mostrou-se causa de morte $58,9 \%$ mais comum que nos Estados Unidos em 2000²1.

Diabetes e hipertensão arterial são fatores de risco para outras doenças circulatórias, reduzindo a qualidade de vida e onerando o sistema de saúde público e privado. Em estudos de prevalência realizados entre idosos, as doenças mais comuns foram a hipertensão arterial e o diabetes. A prevalência da hipertensão chegou a superar 33,3\% dos idosos e o diabetes aproximou-se 
dos 20\%, como entre os idosos institucionalizados de Pelotas (RS) ${ }^{37}$. Em 2006, em São Paulo (SP), 62,3\% dos idosos autorreferiram hipertensão e $21,5 \%$ diabetes ${ }^{6}$.

Nesse estudo, a maior ocorrência de mortes por causas externas, no sexo masculino, corrobora com o observado no país: em todas as idades e em todos os estados da federação, é sempre maior a mortalidade por atropelamentos no sexo masculino $^{38}$. No geral, os homens são as principais vítimas dos acidentes e violências, numa razão que chega a 12 para $1^{39}$ e o consumo pesado de álcool, que é um fator de risco para acidentes e violências diversas, mostrou-se 10 vezes maior em homens que em mulheres em Pelotas ${ }^{15}$.

Este estudo da mortalidade de idosos em Araraquara (SP), no período de 2006 a 2011, preten- deu oferecer um panorama de sua ocorrência, trazendo subsídios para a elaboração de políticas públicas que levem à melhoria da atenção à saúde dessa faixa etária.

\section{Colaboradores}

R Telarolli Júnior trabalhou na concepção e na discussão dos resultados e LCM Loffredo fez o delineamento metodológico e analisou os dados, tendo ambos participado igualmente da redação final do artigo.

\section{Referências}

1. Ramos LR, Veras RP, Kalache A. Envelhecimento populacional: uma realidade brasileira. Rev Saude Publica 1987; 21(3):211-214.

2. Lloyd-Sherlock P. Population ageing in developed and developing regions: implications for health policy. Soc Sci Med 2000; 51(6):887-895.

3. Instituto Brasileiro de Geografia e Estatística (IBGE). Censo demográfico 2010. [Internet]. Rio de Janeiro: IBGE; 2012. [acessado 2012 jun 12]. Disponível em: http://www.censo2010.ibge.gov.br/amostra

4. Telarolli Júnior R, Machado JCMS, Carvalho F. Perfil demográfico e condições sanitária dos idosos em área urbana do Sudeste do Brasil. Rev Saude Publica 1996; 30(5):485-496.

5. São Paulo. Fundação Sistema Estadual de Análise de Dados. Projeções populacionais: 2000 a 2020 [Internet]. São Paulo: SEADE; 2012. [acessado 2012 maio 1]. Disponível em: http://www.seade.gov.br/ produtos/projpop

6. Lebrão ML, Duarte YAO, Santos JLF, Laurenti R. Evolução das condições de vida e saúde da população idosa do Município de São Paulo. São Paulo Perspec 2008; 22(2):30-45.

7. Góis ALB, Veras RP. Informações sobre a morbidade hospitalar em idosos nas internações do Sistema Único de Saúde do Brasil. Cien Saude Colet 2010; 15(6):2859-2869.

8. Brasil. Sistema de Informação de Agravos de Notificação (Sinan). Brasília: Ministério da Saúde (MS); 2012 [Internet]. [acessado 26 maio 2012]. Disponível em: http://dtr2004.saude.gov.br/sinanweb/index.php

9. Dever GEA. A epidemiologia na administração dos serviços de saúde. São Paulo: Pioneira; 1988.
10. Ministério da Saúde (MS).Sistema de Informações sobre Mortalidade (SIM). Brasília: MS; 2011. [Internet]. [acessado 26 maio 2012]. Disponível em: http:/ /svs.aids.gov.br/cgiae/sim/

11. Mello Jorge MHP, Laurenti R, Gotlieb SLD. Análise da qualidade das estatísticas vitais brasileiras: a experiência de implantação do SIM e do SINASC. Cien Saude Colet 2007; 12(3):643-654.

12. Mello Jorge MHP, Laurenti R, Nubila, HBV. O óbito e sua investigação. Reflexões sobre alguns aspectos relevantes. Rev Bras Epidemiol 2010; 13(4): 561-576.

13. Abreu DMX, Sakurai E, Campos LN. A evolução da mortalidade por causas mal definidas na população idosa em quatro capitais brasileiras, 1996-2007. Rev Bras Est Pop 2010; 27(1):75-88.

14. Teixeira INAO, Guariento ME. Biologia do envelhecimento: teorias, mecanismos e perspectivas. Cien Saude Colet 2010; 15(6):2854-2857.

15. Campolina AG, Dini PS, Ciconelli RM. Impacto da doença crônica na qualidade de vida de idosos da comunidade em São Paulo (SP, Brasil). Cien Saude Colet 2011; 16(6):2919-2925.

16. Geib LTC. Determinantes sociais da saúde do idoso. Cien Saude Colet 2012; 17(1):123-133.

17. Magalhães APR, Paiva SC, Ferreira LOC, Aquino TA. A mortalidade de idosos no Recife: quando o morrer revela desigualdades. Epidemiol Serv Saude 2011; 20(2):183-192.

18. Moraes SA, Suzuki CS, Freitas ICM, Costa JrML. Mortalidade por doenças do aparelho circulatório no município de Ribeirão Preto-SP, de 1980 a 2004. Arq Bras Cardiol 2009; 93(6):637-644. 
19. Fratezi FR, Gutierrez BAO. Cuidador familiar do idoso em cuidados paliativos: o processo de morrer no domicílio. Cien Saude Colet 2011; 16(7):3241-3248.

20. Lima-Costa MF, Peixoto SV, Giatti L. Tendências da mortalidade entre idosos brasileiros (1980-2000). Epidemiol Serv Saude 2004; 13(4):237-246.

21. Rebouças M, Pereira MG. Indicadores de saúde para idosos: comparação entre o Brasil e os Estados Unidos. Rev Panam Salud Publica 2008; 23(4): 237-246.

22. Belon AP, Barros MBA. Esperança de vida ao nascer: impacto das variações na mortalidade por idade e causas de morte no Município de Campinas, São Paulo, Brasil. Cad Saude Publica 2011; 27(5):877-887.

23. Carmo CN, Hacon SS, Jacobson LSV, Mourão DS, Ignotti E. Mortalidade por doenças cardiorrespiratórias em idosos no estado do Mato Grosso do Sul, 1986 a 2006. Rev Saude Publica 2010; 44(6):1112-1119.

24. Veras RP. Experiências e tendências internacionais de modelos de cuidado para com o idoso. Cien Saude Colet 2012; 17(1):231-238.

25. Campagna AS, Dourado I, Duarte EC, Daufenbach LZ. Mortalidade por causas relacionadas à influenza em idosos no Brasil, 1992 a 2005. Epidemiol Serv Saude 2009; 18(3):209-218.

26. Oliveira-Campos M, Cerqueira MBR, Rodrigues Neto JF. Dinâmica populacional e o perfil de mortalidade no município de Montes Claros (MG). Cien Saude Colet 2011; 16(Supl. 1):1303-1310.

27. São Paulo. Centro de Vigilância Epidemiológica "Prof. Alexandre Vranjac". Campanhas de vacinação contra influenza por ano-coberturas por município e série total do estado, 2006 a 2008. (Série histórica e outras informações). [página na Internet]. [acessado 2012 maio 25]. Disponível em http://www.cve. saude.sp.gov.br/htm/imuni/imuni_dados.html

28. Maia FOM, Duarte YAO, Lebrão ML, Santos JLF. Fatores de risco para mortalidade em idosos. Rev Saude Publica 2006; 40(6):1049-1056.

29. Dip RM, Cabrera MAS. Vacinação contra a gripe em idosos não-institucionalizados: estudo de base populacional em município de médio porte do sul do Brasil. Cad Saude Publica 2010; 26(5):1035-1044.

30. Francisco PMSB, Barros MBA, Cordeiro MRBB. Vacinação contra influenza em idosos: prevalência, fatores associados e motivos da não-adesão em Campinas, São Paulo, Brasil. Cad Saude Publica 201; 27(3):417-426.
31. Burns VE, Ring C, Carroll D. Factors influencing influenza vaccination uptake in an elderly community-based sample. Vaccine 2005; 23(27):3604-3608.

32. Landi F, Onder G, Carpenter I, Garms-Homolova $\mathrm{V}$, Bernabei R. Prevalence and predictors of influenza vaccination among frail, community-living elderly patients: an international observational study. Vaccine 2005; 23(30):3896-3901.

33. Lima-Costa MF. Fatores associados à vacinação contra gripe em idosos na região metropolitana de Belo Horizonte. Rev Saude Publica 2008; 42(1):100107.

34. Costa MMF, Guerra HL, Barreto SM, Guimarães RM. Diagnóstico da situação de saúde da população idosa brasileira: um estudo da mortalidade e das internações hospitalares públicas. Informe Epidemiologico SUS 2000; 9(1):23-41.

35. São Paulo. Fundação SEADE. Tendências da mortalidade da população paulista por neoplasias malignas. SP Demográfico 2007: 8(3).

36. Sakata S. Diabetes Mellitus entre os idosos no Município de São Paulo: uma visão longitudinal [dissertacão]. São Paulo: Universidade de São Paulo; 2006.

37. Carvalho MP, Luckow ELT, Siqueira FV. Quedas e fatores associados em idosos institucionalizados no município de Pelotas (RS, Brasil). Cien Saude Colet 2011; 6(6):2945-2952.

38. São Paulo. Fundação SEADE. Mortalidade por atropelamento - São Paulo acompanha a média nacional, que é três vezes maior que a dos países do primeiro mundo. SP Demografico 2006; 7(3).

39. Minayo MCS. Seis características das mortes violentas no Brasil. Rev Bras Est Pop 2009; 26(1):135140 .

Artigo apresentado em 02/07/2012

Aprovado em 31/07/2012

Versão final apresentada em 07/08/2012 